\title{
A robust surface matching technique for coastal geohazard
}

\section{assessment and management}

Dr Pauline Miller, Prof. Jon Mills ${ }^{1}$, Dr Stuart Edwards

School of Civil Engineering \& Geosciences

Newcastle University

Newcastle upon Tyne

NE1 7RU

United Kingdom

Paul Bryan

English Heritage Metric Survey Team

Tanner Row

York

Y01 6WP

United Kingdom

Dr Stuart Marsh

British Geological Survey

Keyworth

Nottingham

NG12 5GG

United Kingdom

Dr Harvey Mitchell

School of Engineering

University of Newcastle

Callaghan NSW 2308

Australia

\section{Abstract}

${ }^{1}$ Corresponding author: j.p.mills@ncl.ac.uk; telephone: +44 (0)191 222 5393; fax: +44 (0)191 2226502 
Coastal change is a major issue in many regions of the world, and is often driven by geohazard processes such as landslides and rockfalls. Effective assessment of such phenomena is essential for successful management of coastal ecosystems, and is often reliant on GIS-based analysis. However, while it is crucial that multi-temporal datasets can be accurately registered to a common reference system, traditionally, the dynamic nature of the coastal environment has hampered this process. This paper presents a robust surface matching technique which overcomes the requirement for physical control points, and instead derives control directly from the DEM surfaces. Although surface matching procedures are well established, performance can be sub-optimal where the surfaces contain regions of difference, such as those associated with geohazard activity or vegetation effects. The crucial aspect of the least squares matching approach developed here, is the incorporation of a robust estimation function which allows the effects of surface discrepancies to be mitigated through outlier handling. Aerial photogrammetry is an established technique for coastal monitoring, and extensive archival collections exist. However, archival datasets are particularly affected by the difficulties associated with acquisition of ground control. Conversely, the maturing technique of airborne laser scanning is less influenced by such problems, and instead is capable of producing a high quality representation of coastal terrain. This paper describes the application of the robust surface matching technique to test sites located on the east coast of England.

Photogrammetric DEMs are approximately oriented, before being matched to control surfaces derived from higher order datasets, including airborne laser scanning DEMs. The robust matching algorithm is shown to produce significantly improved results over ordinary surface matching. Analysis indicates the effectiveness of this technique for exploitation of archival datasets, revealing a signature of extensive geohazard activity over the twenty-five year study period. Robust matching of airborne laser scanning datasets has also enabled the quantification of short-term geohazard activity, demonstrating the flexibility of this strategy.

\section{Keywords}

surface matching; airborne laser scanning; lidar; photogrammetry; coastal management. 


\section{Introduction}

Coastal change presents a major concern in many regions of the world. With two-thirds of the world's population occupying the coastal margins, and this figure projected to continue to increase in the future (World Bank, 1996), there is real concern as to the capacity of coastal ecosystems to adjust to the pressures imposed by increased levels of development. In this context, coastal geohazards represent a major driver for coastal evolution, and include processes such as landslides, mudflows and rockfalls, which often occur where the incidence of land and sea results in cliffed terrain. Despite the fact that individual events may be relatively insignificant, the cumulative effects can be dramatic (Lee et al., 2001). Parts of the east coast of England are particularly susceptible to recession, being composed of weak glacial tills and poorly-consolidated materials. One of the most spectacular and celebrated examples of recent times was the 1993 Holbeck Hall Hotel landslip on the North Yorkshire coast, where $60 \mathrm{~m}$ of cliff collapsed overnight, and a further $35 \mathrm{~m}$ was lost over the following days (Lee and Clark, 2002).

Effective assessment of coastal geohazards is vital for successful coastal management, and contributes to wider analysis of coastal change. This process is also essential for furthering understanding of coastal geohazards, particularly in relation to the underlying mechanisms which drive their development. This includes a requirement for accurate datasets which can support quantitative analysis of change in a manner which is both temporally and spatially appropriate. GIS is commonly utilised in coastal management, enabling effective reconciliation of multiple datasets, and providing a platform for a range of analytical tasks. GIS also provides a means of evaluating how such processes interact with coastal ecosystems, impacting on vegetation communities and aspects of the physical environment. However, the quality of the derived results is highly dependant on the input datasets, and one of the most fundamental concerns relates to accurate registration. Registration errors have the potential to propagate into subsequent change analysis, including volumetric calculations, and cliff recessions rates. This issue is particularly important where 
datasets from a number of different sources and temporal epochs are be being analysed, and therefore, is of prime concern in geohazard assessment.

The utilisation of up-to-date datasets is paramount in assessing coastal change, as these enable timely evaluation of existing and developing geohazards. However, it is also important that such information is placed in the appropriate historical context. While an over-reliance on the use of historical datasets should be avoided, such information often forms a necessary input for numerical modelling of future cliff evolution (Hall et al., 2002), and is necessary for the derivation of cliff recession rates. However, the coastal zone presents a dynamic and hostile environment, which has generally proven unreceptive to the application of monitoring techniques, both terrestrial and airborne. Tidal conditions exert significant restrictions on the length of available, continuous survey time, and can prove problematic for airborne monitoring approaches where acquisition at low tide is generally preferable. The flaws of traditional surveying approaches are often related to the fact that they tend to be rather crude and spatially restricted (Hapke and Richmond, 2000), for example, by utilising methods such as total station profile surveys. Such techniques are also labour intensive, and require prolonged, direct contact with potentially hazardous terrain.

Airborne laser scanning (ALS), also referred to as lidar, offers fast, remote capture of mass point cloud data, and as noted by Ackermann (1999) enables the generation of digital terrain models in a highly automated manner. While photogrammetry may suffer from image correlation difficulties in areas such as beaches, or regions of deep shadow (Hapke and Richmond, 2000), as a direct sensing technique, ALS is not affected by such issues, and instead is capable of producing a rapid, reliable representation of coastal terrain (Würländer et al., 2004). Although ALS is still maturing as a technology, it facilitates effective acquisition over wide areas, and holds tremendous promise for coastal monitoring applications, as demonstrated through a number of investigations (e.g. Saye et al., 2005; Shrestha et al., 2005). However, as this technique has only become widely available relatively recently, it is unable to provide information on longer term landscape change. 
Aerial photography is an established technique which has been effectively utilised in assessment of coastal change for decades. Consequently, in many regions of the world, a rich archive of aerial imagery exists. This often represents the most accessible source for quantification of coastal change over the last fifty to sixty years. Brown and Arbogast (1999) investigated changes to sand dunes on the shores of Lake Michigan, USA, by comparing DEMs derived from stereo photography from 1965 and 1987 . While the quality of their results was limited by the use of paper prints, and low accuracy ground control points, their findings emphasised the potential of the archival photogrammetric technique for retrospective assessment of dynamic coastal terrain. Adams and Chandler (2002) provide an example which evaluates changes to a coastal mudslide on the south coast of the UK, through the use of aerial photography from 1976, and an ALS dataset acquired in 1998, indicating the potential of this approach for assessment of coastal geohazard activity. However, one of the main barriers to successful photogrammetric exploitation of archival aerial imagery, is the difficulty associated with establishing suitable ground control (James et al., 2006). Although recent developments in on-board position and orientation systems for direct orientation of imagery may have reduced control requirements, this still remains a major issue for archival datasets, particularly in coastal environments, which can be relatively featureless in this regard.

The aim of the research presented here is to develop an efficient strategy for coastal geohazard assessment, which is capable of effectively addressing the essential and often troublesome dataset registration task. Ultimately, this will facilitate improved understanding of coastal geohazard processes, enabling more effective coastal management. In order to fulfil this aim, this paper describes the development of a surface matching technique which is capable of integrating multitemporal datasets. This approach is based on an algorithm which performs least squares minimisation of vertical surface differences in order to determine the unknown transformation parameters required to align overlapping DEMs. The incorporation of robust functionality mitigates the contaminating effects of surface discrepancies introduced through geohazard activity, or vegetation change (particularly where vegetation effects have only been partially removed through 
filtering). In addition to solving the essential registration task, the software possesses an inherent capacity for difference detection, thus providing an elegant and cost-effective solution for coastal geohazard monitoring. The strategy is evaluated through application to two test sites located on the North Yorkshire coast of eastern England.

\section{Methodology}

\section{1. $\quad$ Surface Matching}

\subsubsection{Background}

Cooper (1998) notes that changes to coordinates over time arise not only because of changes to the terrain itself, but also as a result of measurement errors and datum differences, and that as only the first effect is of interest in monitoring applications, it is necessary to minimise the effects of the latter. Any registration error introduced at this early stage can propagate into subsequent analysis, with the potential to contaminate measures of volumetric change, cliff recession rates, the interpretation of geohazard behaviour, and any ensuing actions based on such information. In this research, a software-based solution has been adopted in order to address the vital registration issue. This approach is based upon least squares surface matching, a technique which enables a poorlycontrolled matching surface to be registered to a well-controlled reference surface. This can also be considered as a method for transforming a DEM from some arbitrary local coordinate system to a reference or global coordinate system. It is important to note that the reference surface is held fixed, while the matching surface (which can be considered as a 'floating' surface), is fitted to this. The solution is founded on the standard 3D conformal coordinate transformation, which defines the three rotations $(\omega, \phi, \kappa)$, three translations $\left(T_{\mathrm{x}}, T_{\mathrm{y}}, T_{\mathrm{z}}\right)$ and scale factor $(s)$ required to relate two sets of known points in separate coordinate systems (Wolf and Dewitt, 2000). However, for the reasons already discussed, it may prove difficult to identify suitable control points in the coastal context, and so surface matching can be applied to overcome this requirement for physical control points. 
Instead, each point on one surface effectively provides a control point in height (Ebner and Strunz, 1988). In this manner, the algorithm utilises conjugate surface patches, by attempting to globally minimise the sum of the squares of the vertical differences between the points on the matching surface, and corresponding surface patches on the reference surface (Mills et al., 2003). As the problem is non-linear, good initial approximations are required for the unknown transformation parameters, and the solution is iterated until convergence. In the algorithm utilised here, the reference standard deviation and the parameter corrections (used to update the initial approximations for the unknown transformation parameters) are monitored from iteration to iteration, and convergence is achieved when these values change by an insignificant amount.

In addition to addressing the registration issue, surface matching also provides two further important advantages. Firstly, it offers an inherent capacity for change detection, as the post-match surface residuals indicate areas of difference between the two surfaces, which may be due to terrain change. Secondly, the surface matching procedure provides an elegant means of identifying and eliminating systematic error which may exist in the matching surface, as demonstrated by Maas (2002) in relation to offsets between overlapping ALS strips. This paper utilises an algorithm which originated at the University of Newcastle, Australia (Mitchell, 1994), and which was later developed for application to irregular topographic datasets in the context of a coastal monitoring research project, as described by Mills et al. (2003, 2005).

It is beyond the scope of this paper to elaborate on the mathematical formulation of least squares surface matching through vertical distance minimisation, which is well developed and documented, with minor variations, by Karras and Petsa (1993); Mitchell and Chadwick (1999) and Rosenholm and Torlegård (1988). While initial algorithms were designed for application to grid-based datasets, recent efforts have focussed on irregular topographic DEMs, including those derived from ALS and photogrammetry (e.g. Maas, 2002; Mills et al., 2003). Topographic surfaces such as these can generally be described as 2.5D, and as Mitchell and Chadwick (1999) observe, this is a scenario which is highly favourable for minimisation of vertical differences, and which does not warrant the 
implementation of more complex approaches such as the iterated closest point (ICP) algorithm (refer to Besl and McKay, 1992). It should be noted that the complexity of the ICP approach stems from the exhaustive search for 3D point correspondence, which enables determination of the optimum transformation aligning the surfaces in 3D space. Where surfaces contain strong vertical components (e.g. building facades), fully-3D matching algorithms are often implemented. In addition to the ICP, a number of other 3D approaches, many of which are based upon evaluating the Euclidean distances between the surfaces, have been developed. A review of these approaches is beyond the scope of this paper, but a comprehensive overview of these and other strategies can be found in Gruen and Akça (2005). In the context of this research, although the coastal cliffs present some vertical elements, a fully-3D solution is not required, as the matching surfaces are primarily composed of undulating cliff-top topography.

However, the existing matching algorithm requires further optimisation for application to coastal geohazard datasets. The main complication relates to the fact that in the context presented here, the surfaces to be matched will never be identical. As Pilgrim (1996) observes, differences can arise for a number of reasons, including as a result of time-induced changes, or where the surfaces have been captured through different techniques. With reference to the former situation, geohazard activity, or vegetation change, has the potential to introduce significant discrepancies to the matching surfaces, while the latter situation is also of great relevance, as the techniques of ALS and photogrammetry are likely to return differing surface representations, particularly with regards to vegetation representation. This is true, even where vegetation has been removed through filtering algorithms, as there will always be residual effects due to the differing and inconsistent vegetation penetration properties of these two techniques. The introduction of local surface discrepancies will influence the estimation of the transformation parameters, and where the effects are significant, conventional least squares approaches may fail, or may converge to an erroneous solution (Li et al., 2001; Pilgrim, 1996). Although minor differences between the surfaces can be tolerated, the assumption that the surfaces are overwhelmingly similar is critical for attaining a successful solution. Karras 
and Petsa (1993) implemented the surface matching procedure for matching of close range photogrammetric surfaces for deformation detection in medical applications, and in recognition of the contaminating influence of outlier observations, applied a data snooping technique to eliminate gross errors. However, this approach is only capable of removing a single outlier at a time. Pilgrim (1996) has carried out investigations into overcoming outlier contamination through the development of a robust form of least squares surface matching. This incorporates a weighting function based on a modified maximum likelihood estimator (M-estimator), which identifies, and down-weights high-residual observations which can effectively be considered as outliers in the matching. This strategy was further investigated by Li et al. (2001). However, these studies were motivated by medical and industrial applications, and although they demonstrated the superior results which could be obtained through a robust approach, both studies were restricted to simulated datasets. The application presented in this paper provided an excellent opportunity to investigate the potential of robust surface matching for effective registration and difference detection in relation to topographic datasets, and the development of this algorithm is detailed in the following section.

\subsubsection{Robust Surface Matching Development}

The inclusion of a weight matrix is a standard extension to least squares, and is widely used in order to reduce the contribution of less reliable observations (Draper and Smith, 1998). This is a core aspect, as robust functions can be incorporated in the existing algorithm through the technique of iteratively reweighted least squares (IRLS). Robust matching draws from robust estimation theory, which is defined by Rousseeuw and Leroy (1987) as relating to regression techniques which are resistant to the contaminating effects of outlying observations. In essence, weights are derived from a weighting scheme, which is dependant on the choice of robust estimation function. This enables those observations which produce large residual values to be down-weighted accordingly (Draper and Smith, 1998). A comprehensive overview of the influence of outliers and robust regression theory can be found in a number of texts, including Draper and Smith (1998) and Rousseeuw and 
Leroy (1987). M-estimators are a popular family of robust estimators, offering flexible performance (Goodall, 1983), and permitting straightforward incorporation in least squares routines. Specifically, Tukey's Biweight function has been utilised here. The Biweight is one of the most commonlyutilised M-estimators, and as noted by Goodall (1983) is difficult to surpass in terms of delivering good overall performance in most situations. The weight function for the Biweight is defined as follows:

$$
w_{b}(u)= \begin{cases}\left(1-u^{2}\right)^{2} & |u| \leq 1 \\ 0 & |u|>1\end{cases}
$$

Where the weights, $w_{b}$, ranging from 0 to 1 , are calculated from $u$, the standardised least squares residuals, following each iteration of the software. As defined here, the weighting scheme is optimised for efficiency at the normal distribution. Although this may lead to a large portion of the observations being weighted out of the matching procedure, the DEMs contain a great deal of redundancy. Consequently greatest emphasis is placed on low-residual observations, which are most likely to correspond to stable regions (assuming that the surfaces are in good initial alignment). The weighting function is incorporated through IRLS, proceeding as follows (Wager et al., 2005):

1. select a suitable robust weighting scheme (the Biweight in this case);

2. obtain initial residuals from un-weighted least squares (first iteration);

3. apply the M-estimator weight function to compute the robust weights, and place these on the diagonal of the least squares weight matrix;

4. re-evaluate the least squares estimation (next iteration) using the new weights to compute updated residuals;

5. repeat steps 3 and 4 until convergence.

Robust least squares implemented through IRLS provides a mechanism for automated outlier handling, where weights are not held fixed, but alter from iteration to iteration in response to the 
changing residuals. The existing algorithm was further developed to incorporate this robust capacity, and initial testing was carried out using artificial datasets with simulated outlier effects. This testing verified the integrity of the algorithm, and demonstrated improved performance over the un-weighted version where concentrated regions of outliers were present. Details of this testing can be found in Miller et al. (2007).

\section{2. $\quad$ Test Sites}

Test sites for this research are located on the North Yorkshire coast of eastern England (Figure 1), which provides an excellent arena for evaluation of the monitoring strategy, as it offers a range of active geohazard processes. The first test site is located at Filey Bay, which lies approximately eleven kilometres south of the resort town of Scarborough. Although erosion rates in this area are relatively low, locations prone to coastal landslips are eroding more rapidly (by up to $2 \mathrm{~m}$ per year), and in a more irregular manner (Capita Symonds, 1998). The ten kilometre-long bay is fronted by a broad, flat sandy beach, and is backed by moderately steep, soft glacial till cliffs which rise to between $30 \mathrm{~m}$ and $50 \mathrm{~m}$ in height, as illustrated in Figure 2a. The cliffs are variable in profile, and interspersed with vegetation, mainly in the form of rough grass.

The second test site is located some 40 kilometres north, at Whitby Abbey Headland (Figure 2b), where near-vertical cliffs rise to around $60 \mathrm{~m}$ in height. The geology of weak rocks and glacial tills is prone to erosion, not only through wave attack, but also due to the effects of groundwater seepage from within the cliffs (Clark and Guest, 1991). Geohazard processes at this location pose a serious risk to important infrastructure, such as the coastguard station, located on the clifftop, and also threaten areas of the town itself. Furthermore, cliff erosion has impacted upon the nearby $7^{\text {th }}$ century Whitby Abbey site, where action has already been required in order to prevent valuable archaeology from being lost to the sea following a major cliff failure in 2000. As Figure 2b illustrates, the Whitby site is partly protected by a 4 metre high rock armour revetment, which was installed in 2000 to combat the effects of marine erosion at the cliff toe (Rosser et al., 2005). Whereas the soft 
glacial tills of Filey Bay are characterised primarily by landslides, Whitby is affected by rockfalls of varying magnitudes.

\subsection{Data Acquisition and Pre-Processing}

The UK Natural Environment Research Council's Airborne Research and Survey Facility (NERC ARSF) acquired ALS data for both test sites in April 2005, August 2005 and May 2006. The data were captured from a flying height of 1000 m, using an Optech ALTM 3033 instrument (first and last pulse return), mounted on a Dornier 228-101 research aircraft. This translated to a ground resolution of approximately 1 point $/ \mathrm{m}^{2}$. The data were georeferenced by the ARSF prior to being delivered by flightline in ASCII $x y z$ format. Terrasolid's TerraScan software was used in order to refine the ALS point cloud prior to surface matching. The first pulse returns were discarded, and the last pulse return data were filtered using the TerraScan ground classification routine. This enabled the removal of vegetation and building points, thus ensuring that change analysis would be primarily focussed on terrain processes. The TerraScan ground classification routine has been reported to offer good performance over areas of steep slopes (Sithole and Vosselman, 2003), and visual inspection suggested that the resultant terrain models were of high quality. However, it is important to acknowledge that some vegetation effects will always remain, particularly in regions of dense vegetation, where the laser signal may have been unable to fully penetrate to the ground surface.

As discussed in Section 1, aerial photography is commonly used for extracting quantitative change information in coastal studies. Archival aerial photographs dating back to 1980 were acquired for the study sites, with details as indicated in Table 1. With the exception of the 1994 photography, which had already been processed by English Heritage, the imagery was photogrammetrically scanned and then processed in a SocetSet digital photogrammetric workstation. Following relative orientation, an approximate absolute orientation was performed using control points derived from large scale mapping, and $1 \mathrm{~m}$ DEMs were then extracted for the study sites. Further processing was 
carried out in order to classify and remove non-ground points. Although TerraScan is designed for processing of laser scanning datasets, the photogrammetric DEM is not dissimilar to ALS data, and so, for consistency, TerraScan was again utilised for ground classification, as described previously. A number of check point datasets were available for evaluating the quality of the results. For the Filey Bay test site, 845 check points, acquired through kinematic GPS, were available for a hardsurfaced, level road circuit. The standard deviation of the dataset was $0.018 \mathrm{~m}$ in height, and although no absolute measure of accuracy was available, this was anticipated to be in the region of $0.01 \mathrm{~m}$. Ten GPS ground control points (surveyed by static GPS) were also acquired for the area of Filey Bay covered by the archival aerial photography, so that a conventional absolute orientation could be performed for validation purposes. At the Whitby test site, a number of check points were available. These had been measured by static GPS for topographic surveys of the headland in 1994 and 1999, and were located at permanent survey stations, well distributed across stable parts of the headland.

\section{Results}

\subsection{Geohazard Assessment at Filey Bay}

The robust matching algorithm was applied to multi-temporal datasets at the Filey Bay test site in order to evaluate the monitoring strategy, and its ability to accurately determine geohazard change. A test area at the southern end of Filey Bay was selected for analysis. This site includes a relatively extensive landslide complex (Figure 3), which lies adjacent to a large caravan park. Visual inspection of the historical imagery indicated that the site was relatively active in terms of geohazard activity, and therefore, it was anticipated that this should provide a good opportunity for rigorous evaluation of the robust matching algorithm.

The 1980 epoch of stereo aerial photography, which had been approximately oriented, was selected as the matching surface, and the April 2005 ALS dataset provided the fixed reference surface; for 
the purposes of this investigation, it was assumed that the ALS dataset would offer superior control, as this had been directly georeferenced through in-flight GPS/INS. The ALS data were compared to the GPS check data for the road circuit. This confirmed that the vertical accuracy was within the manufacturer's specification of $\pm 0.15 \mathrm{~m}$. The robust surface matching software was then used to match the 1980 photogrammetric DEM to the 2005 ALS control surface, and this procedure was repeated using the un-weighted matching algorithm. In both cases, the software was applied in full seven parameter mode (3D conformal coordinate transformation). Matching was carried out over the area covered by the 1980 DEM, which included an extensive area of cliff-top and the cliff face. Although the robust estimator is more tolerant to change than un-weighted least squares, it is important to ensure that the discrepancies do not overwhelm the surface area, and therefore inclusion of the relatively stable cliff top area was essential.

Examination of the matching solutions indicated that slightly different transformations had been obtained by the two least squares matching algorithms. This was confirmed by plotting the postmatch residuals, which correspond to final surface differences between the 1980 and 2005 DEMs (Figure 4). From examination of these, it can be seen that slightly different patterns of change have emerged through the two approaches. The robust matching shows net erosion over most parts of the cliff-face, a trend which would fit with natural expectation, but which is not so obvious in the unweighted results. This is highlighted by the results at areas A and B (Figure 4), where predominantly negative change is apparent in the robust solution, but in contrast, largely positive change has occurred in the un-weighted results. It is likely that in standard least squares fashion, the un-weighted algorithm has adjusted the position of the 1980 DEM to best 'balance out' the differences between the two surfaces, irrespective of whether or not such discrepancies relate to actual terrain change. Consequently, in the un-weighted results, areas of positive and negative change have been more equally adjusted across the cliff-face than for the robust matching. Both matching solutions clearly highlight development of the landslide which is located at the extreme south-eastern end of the test area, and which is bisected by the northern end of profile 3 (Figure 4). 
This failure, also evident in Figure 3, appears to have widened over the twenty-five year period, as indicated by net erosion of the side scarps. The robust solution also suggests the presence of a subtle patchwork of change over the western portion of the cliff-top. At first glance this may seem anomalous, but in fact, it can be attributed to the caravans (mobile homes) which occupy this portion of the caravan park. Between 1980 and 2005, the distribution of these structures has altered, and although a ground classification was carried out on both DEMs prior to matching, it is likely that the photogrammetric DEM in particular would have returned a poor representation of the ground surface, given the tightly packed nature of these structures, and the $1 \mathrm{~m}$ TIN representation. Consequently, it is to be anticipated that a pattern of change such as this will arise. However, it is interesting to note that this is not so pronounced in the un-weighted matching residuals, a further example of the un-weighted algorithm balancing the differences between the surfaces. It should be noted that the large area of positive change which appears in the extreme western corner of both surfaces corresponds to the construction of the caravan park leisure complex.

The next stage involved quantitative assessment of the quality of the matching results. The ten GPS ground control points (GCPs) located in the stereo overlap region, were used in photogrammetric processing to perform a rigorous absolute orientation of the 1980 stereomodel. Following this, it was possible to extract a validation DEM, which represented the conventional control point-based registration approach, and effectively provided a benchmark for evaluation of the matching results. The two post-match, transformed 1980 surfaces were then compared to the 1980 validation surface by analysing vertical surface separations along the profiles indicated in Figure 4. Figure 5 illustrates the root mean square error (RMSE) for each profile. These results highlight the superior registration of the robust matching solutions, which in general appears twice as accurate as the un-weighted matching. However, it is also important to acknowledge that there is still some discrepancy between the robust solution and the validation surface. The most likely reason for this is that the April 2005 ALS surface was used as the reference in the matching. This will have resulted in the 1980 matching DEMs being transformed to slightly different positions than that of the 1980 validation 
DEM. Furthermore, the largest RMSE values occur in profiles which include significant portions of the cliff face, where the effects of slope and vegetation are likely to exacerbate any discrepancies.

\subsection{Geohazard Assessment at Whitby Abbey Headland}

After establishing the integrity of the robust matching algorithm, this strategy was applied for multitemporal assessment at the Whitby test site. Initial analysis of the available datasets revealed that in this case, the 1994 photogrammetric DEM offered the highest accuracy. This $1 \mathrm{~m}$ grid-structured DEM was produced by the English Heritage Metric Survey Team for a 3D mapping project, and was derived from large scale aerial photography $(1: 1600)$ which had been rigorously controlled by GPS GCPs. Given the large scale of this photography and the professional nature of the product, it was anticipated that this would provide the most suitable control surface. The 1986 DEM (approximately oriented), and the ALS DEMs from April 2005, August 2005, and May 2006 were then individually matched to the 1994 reference surface in both robust and un-weighted modes. This process was slightly more challenging than the matching of the Filey datasets. The main difficulty related to the relatively flat terrain of the cliff-top, which in some cases lacked the surface gradients required to constrain the matching solution. However, when this particular difficulty was encountered, it was successfully overcome by retaining buildings in the DEMs.

Upon completion of the surface matching, the quality of the solutions was analysed through comparison with the GPS check point dataset. For each match, both the pre-match and post-match (robust and un-weighted) matching surfaces were compared to the check points in order to determine the effectiveness of surface matching in improving absolute orientation. Furthermore, the quality of the reference surface, the 1994 DEM, was also assessed, as this provided a measure of the best possible accuracy which could be anticipated, post-match, in all cases. Results of the quality assessment are detailed in Table 2. These illustrate that in each case, the pre-match surface accuracy has been improved through application of surface matching. This is especially notable for the 1986 surface, which was initially only approximately oriented using points acquired from large scale 
mapping. In this case, application of robust surface matching has seen an improvement from an initial RMSE of $3.875 \mathrm{~m}$ to a post-match value of $0.150 \mathrm{~m}$. This figure is approaching the $0.130 \mathrm{~m}$ RMSE of the 1994 reference surface. While it is important to acknowledge that for the 1986 surface, the results were derived from only a small number of check points (due to the restricted coverage of the 1986 DEM), these values still give a valuable indication of the quality of the absolute orientation solution achievable through robust matching. A further key outcome, which supports the findings presented in Section 3.1, is that in each case, robust matching returned a superior solution to un-weighted matching. In the most extreme example of this, the un-weighted algorithm failed to achieve a solution for the August 2005 dataset, whereas the robust software returned satisfactory results. Although un-weighted matching has produced lower mean values in nearly every case, analysis of the raw residuals for the check points indicates that this is a numerical anomaly; the RMSE values confirm the superiority of the robust solution in all cases.

Following confirmation of the quality of the solutions achieved through robust surface matching, change was analysed for these results in the direction normal to the cliff face. Due to the vertical nature of the cliffs at Whitby, the prime component of retreat is in this direction. Consequently, it was possible to evaluate change across the cliff face in a continuous manner, revealing the extent and nature of geohazard activity over the twenty year period, 1986 to 2006 . The surfaces were compared sequentially in time, in the manner 1986 - 1994, 1994 - April 2005 and so on. Figure 6 illustrates the change to the position of the cliff-top over this time frame. Area A indicates the location of the major cliff failure, referred to in Section 2.2, which occurred in October 2000. It is apparent that intermittent retreat on a lesser scale has also been occurring in the vicinity of the coastguard station and radio mast, both of which represent crucial infrastructure for the town of Whitby. Area B highlights the site of a cliff failure adjacent to the abandoned quarry workings, which affected the upper portion of the cliff face and occurred some time between the August 2005 and May 2006 ALS acquisitions, resulting in a retreat of around $5 \mathrm{~m}$ of the cliff edge. This is illustrated in greater detail in Figure 7, which depicts changes across the cliff face between August 
2005 and May 2006. The failure and associated debris accumulation zone are highlighted on the left, and were confirmed through a subsequent field visit. Volumetric analysis of this failure revealed that $6198 \mathrm{~m}^{3}$ of material had been lost from the cliff face, and $2645 \mathrm{~m}^{3}$ deposited, resulting in a net loss of $3553 \mathrm{~m}^{3}$. This suggests that some material may have been removed through wave action, which is certainly likely, as this is a major mechanism for erosion at Whitby.

\section{Discussion}

Validation of the surface matching results at Whitby returned particularly good results, no doubt influenced in part by the level nature of the terrain here. However, this has demonstrated the quality of the solutions achievable through surface matching. In general, the vertical accuracies of the robust matching solutions were close to the absolute accuracy of the 1994 reference surface, only differing by a few centimetres. The August 2005 ALS surface displayed the greatest disparity, and failed to converge through un-weighted matching. However, inspection indicated that this surface was particularly noisy, which is the most likely reason for the increased robust RMSE, and unweighted matching failure. The successful matching of the 1986 DEM was particularly encouraging, given that the initial orientation was much poorer than that of the ALS datasets.

The process of validating the Filey Bay matching results reaffirmed the difficulties associated with acquiring suitable ground control points for archival imagery. Despite the fact that the 1980 imagery is of a relatively large scale $(1: 7800)$, the majority of the stereo overlap region contained no suitable hard detail features. Eventually a number of control points were selected in and around the caravan park, but the process of identifying these proved to be extremely time consuming and challenging. The investigations at the Filey Bay site have illustrated the wealth of historical change information often contained in archival datasets, which can be overlooked due to difficulties associated with acquiring suitable ground control. Significant changes were evident over the twenty-five year period, and these were clearly concentrated in a manner consistent with geohazard 
activity. This illustrates the value of robust surface matching for levering the potential of archival datasets, thus facilitating extended monitoring of geohazard behaviour and evolution.

The RMSE of the matching solutions at the Filey Bay test site, determined through comparison to the validation DEM, confirmed that the robust matching algorithm had returned a more accurate registration solution than the un-weighted version of the software. However, it is important to consider the accuracy of the matching solutions in relation to the anticipated magnitude of geohazard activity or more generic change. In this case, the RMSE of the robust solution (refer to Figure 5) is generally not significant in relation to the magnitude of geohazard activity occurring over the cliff-face between 1980 and 2005 (Figure 4). However, over shorter time periods, or in less active settings, the magnitude of detectable terrain change may be limited by the accuracy of the matching technique. Furthermore, although the robust matching algorithm is able to mitigate the effects of local surface differences and outliers, where these effects become too extensive, the performance of the algorithm is likely to become sub-optimal. Further testing and quality analysis are required to fully evaluate and quantify the limitations of the robust matching technique.

This research has been primarily concerned with evaluating robust surface matching as a technique for coastal geohazard assessment, particularly in the context of archival datasets. Consequently, while the selection of a suitably robust function was an integral aspect of the research, this was not the primary focus, and it is important to highlight that alternative functions, even within the M-estimator family, may prove more suitable for other matching scenarios. In this sense, it is important to consider the nature of the datasets, particularly in terms of the extent and nature of any outliers. On the whole, the surface matching approach has demonstrated a great deal of potential. However, in order to achieve a good solution, surface gradients are required (Karras and Petsa, 1993; Mitchell and Chadwick, 1999), and therefore, the geometry of the matching surfaces must be evaluated. This is an intuitive consideration, as without strong surface geometry, the surfaces would be free to slide over each other in an unconstrained manner. This issue arose at the Whitby test site, where matching was carried out over the relatively flat cliff top area. Initial attempts at matching 
over this area failed, or returned weak solutions (large parameter standard deviations, or slow and unconvincing convergence). However, after re-introducing cliff top buildings to the DEMs, improved solutions were achieved, and verified.

The technique of surface matching for absolute orientation of topographic datasets was first introduced to the geomatics community almost twenty years ago, by Ebner and Strunz (1988) and Rosenholm and Torlegård (1988). Although this approach has been revisited intermittently over the last two decades, perhaps wider consideration is now timely, especially given the increasing availability of DEMs of varying accuracies and spatial resolutions, from a range of sensor platforms. However, at present, interest in surface matching remains primarily restricted to terrestrial and close range applications. The research presented here has built upon the findings of Mills et al. (2003) in demonstrating that this approach is equally capable of handling topographic datasets acquired from airborne platforms. Furthermore, it has been shown that DEMs derived from ALS can be successfully utilised to provide control for other datasets. In many parts of the world, and certainly in the UK, ever-increasing swathes of countryside are being acquired through ALS. Although there are still issues associated with the cost of accessing such data, particularly outside of the academic sector, nevertheless, its existence has now become a reality, and as such, it is ripe for exploitation. The potential of ALS data for provision of control information has previously been demonstrated by Habib et al. (2004) and James et al. (2006). The latter extracted ground control points directly from ALS surfaces in order to control archival photogrammetric datasets. However the technique presented here takes this approach forward by utilising the surface geometry in an automated manner. Surface matching offers an elegant solution for dataset registration, with an inbuilt capacity for change detection. This renders it suitable for a range of terrain monitoring scenarios, and particularly relevant to applications such as volcanology or glaciology where the acquisition of control points could otherwise prove hazardous and/or expensive.

Importantly, this paper has highlighted the potential of robust surface matching as a technique for facilitating increased investigation of archival datasets for analysis of coastal change. The research 
presented here has demonstrated the success of this approach for assessment of geohazard activity over localised extents. However, this technique also holds tremendous potential for retrospective analysis over wider extents, enabling identification of large-scale processes influencing coastal change. Archival aerial imagery dating back over fifty years or more provides an accessible record of metric change information in many regions of the world, and coverage is often particularly extensive in coastal regions, as these represent natural borders, and are comprised of important ecosystems. Robust matching provides an efficient mechanism for rigorous exploitation of such datasets, and consequently offers excellent opportunities for retrospective assessment of large-scale processes. Furthermore, as part of a regular monitoring programme, incorporating techniques such as ALS, the approach presented in this paper would enable the development of a reliable and highly automated change detection strategy. Although the surface matching software already offers an automated solution to dataset registration, integration with GIS functionality would facilitate automated analysis and detection of critical change activity. Such an approach could be implemented over relatively large extents, thus offering a practical solution to change monitoring at the coastal management level.

Robust surface matching is also being explored in this research for multi-sensor dataset fusion. This is focussing on the integration of airborne and terrestrial laser scanning datasets in order to investigate a multi-resolution approach to coastal geohazard assessment. The robust matching is able to overcome difficulties associated with the effects of noise in the surfaces, and complications caused by surface vegetation. A multi-temporal, multi-sensor approach is likely to provide the most effective strategy for flexible and efficient coastal monitoring, particularly over large extents, with the surface matching software central to robust reconciliation of the various datasets.

In terms of future research, there are a number of avenues which are worthy of exploration. M-estimators (including the Biweight) generally incorporate one or more tuning constants, which influence the rejection properties of the weight function in order to control robustness to outliers. Given more time, it would be desirable to investigate this aspect in order to optimise robust 
functionality, and evaluate sensitivity to such parameters. Furthermore, it may be useful to explore alternative functions, such as the LMS-estimator (least median of squares), which is recommended by Li et al. (2001) for its excellent robustness qualities. However, the LMS function is not as straightforward to resolve as M-estimator functions, and incorporating this is likely to entail major modifications to the existing algorithm. As discussed above, there is significant potential for increasing the automation of the strategy through integration with GIS, and future research should also concentrate on this aspect.

\section{Conclusions}

In dynamic environments, monitoring, and associated change detection processes are often crucial. In order to facilitate successful GIS-based analysis and derive reliable results, successful dataset registration is essential. Yet the very nature of such regions often precludes this, as it can be difficult to establish the necessary ground control. This is a particular difficulty in coastal regions, where change is often driven by geohazard processes. This paper has developed a robust least squares surface matching technique which is capable of overcoming the requirement for physical control points, instead deriving control information from the surface geometry of DEMs. This utilises a robust function from the M-estimator family, which is incorporated through iteratively reweighted least squares. Crucially, the robust properties enable discrepancies between the matching surfaces to be handled as outlier observations, and their effects mitigated through downweighting. Such discrepancies may arise through the development of geohazard features or vegetation effects. Standard least squares surface matching performance may otherwise become sub-optimal, or may fail altogether if such anomalies are left unaddressed.

In this research, robust surface matching has been investigated for coastal geohazard assessment through application to test sites located on the North Yorkshire coast of eastern England. This has focussed on multi-temporal analysis of DEMs derived from archival photogrammetry and airborne laser scanning. Testing has demonstrated that in the absence of ground control points, airborne laser 
scanning is capable of providing an alternative source of control, thus enabling successful registration of archival photogrammetric datasets. Furthermore, the robust algorithm has been shown to be capable of providing highly accurate dataset registration, and subsequently, the potential of this for quantitative assessment of coastal geohazard activity has been demonstrated. It should be highlighted that this technique is capable of performing the essential dataset registration process, while simultaneously delivering change information through the final matching residuals. Consequently, this strategy offers tremendous potential for a range of applications, including multisensor dataset fusion, and multi-temporal change detection across a range of environments.

\section{Acknowledgements}

This research was jointly funded by English Heritage, the British Geological Survey, and an Engineering and Physical Sciences Research Council (EPSRC) Doctoral Training Grant (EP/P500370/1). The authors are grateful to the Natural Environment Research Council's Airborne Research and Survey Facility for acquisition of airborne laser scanning data, and Ordnance Survey for provision of archival aerial photography.

\section{References}

Ackermann, F., 1999. Airborne laser scanning - present status and future expectations. ISPRS Journal of Photogrammetry and Remote Sensing 54 (2-3), 64-67.

Adams, J.C. and Chandler, J.H., 2002. Evaluation of LIDAR and medium scale photogrammetry for detecting soft-cliff coastal change. The Photogrammetric Record 17 (99), 405-418.

Besl, P.J. and McKay, N.D., 1992. A method for registration of 3-D shapes. IEEE Transactions on Pattern Analysis and Machine Intelligence 14 (2), 239-256.

Brown, D.G. and Arbogast, A.F., 1999. Digital photogrammetric change analysis as applied to active coastal dunes in Michigan. Photogrammetric Engineering and Remote Sensing 65 (4), 467-474.

Capita Symonds, 1998.

http://www.capitasymonds.co.uk/uploaded_files/documents/61_1450_11.pdf [Accessed: 7 th April, 2007].

Clark, A.R. and Guest, S., 1991. The Whitby cliff stabilisation and coast protection scheme. Proceedings of the International Conference on Slope Stability (Institute of Civil Engineers), Thomas Telford, Isle of Wight. pp. 283-290. 
Cooper, M.A.R., 1998. Datums, coordinates and differences. In: Lane, S.N., Chandler, J.H., Richards, K.S. (Eds.), Landform Monitoring, Modelling and Analysis, John Wiley \& Sons, Chichester, pp. 21-35.

Draper, N.R. and Smith, H., 1998. Applied Regression Analysis, third ed. John Wiley \& Sons, New York.

Ebner, H. and Strunz, G., 1988. Combined point determination using digital terrain models as control information. International Archives of Photogrammetry, Remote Sensing and Spatial Information Sciences 27 (Part B11), 578-587.

Goodall, C., 1983. M-Estimators of Location: an Outline of the Theory. In: Hoaglin, D.C., Mosteller, F., Tukey, J.W. (Eds.), Understanding Robust and Exploratory Data Analysis, John Wiley \& Sons, New York, pp. 339-403.

Gruen, A. and Akça, D., 2005. Least squares 3D surface and curve matching. ISPRS Journal of Photogrammetry and Remote Sensing 59 (3), 151-174.

Habib, A.F., Ghanma, M.S., Kim, C.J. and Mitishita, E., 2004. Alternative approaches for utilizing LiDAR data as a source of control information for photogrammetric models. International Archives of Photogrammetry, Remote Sensing and Spatial Information Sciences 35 (Part B1), 193-198.

Hall, J.W., Meadowcroft, I.C., Lee, E.M. and van Gelder, P.H.A.J.M., 2002. Stochastic simulation of episodic soft coastal cliff recession. Coastal Engineering 46 (3), 159-174.

Hapke, C. and Richmond, B., 2000. Monitoring beach morphology changes using small-format aerial photography and digital softcopy photogrammetry. Environmental Geosciences 7 (1), 32-37.

James, T.D., Murray, T., Barrand, N.E. and Barr, S.L., 2006. Extracting photogrammetric ground control from lidar DEMs for change detection. Photogrammetric Record 21 (116), 312-328.

Karras, G.E. and Petsa, E., 1993. DEM matching and detection of deformation in close-range photogrammetry without control. Photogrammetric Engineering and Remote Sensing 59 (9), 1419-1424.

Lee, E.M. and Clark, A.R., 2002. Investigation and Management of Soft Rock Cliffs, Thomas Telford, London.

Lee, E.M., Hall, J.W. and Meadowcroft, I.C., 2001. Coastal cliff recession: the use of probabilistic prediction methods. Geomorphology 40 (3-4), 253-269.

Li, Z., Xu, Z., Cen, M. and Ding, X., 2001. Robust surface matching for automated detection of local deformations using Least-Median-of-Squares estimator. Photogrammetric Engineering and Remote Sensing 67 (11), 1283-1292.

Maas, H.-G., 2002. Methods for measuring height and planimetry discrepancies in airborne laserscanner data. Photogrammetric Engineering and Remote Sensing 68 (9), 933-940.

Miller, P.E., Mills, J.P., Edwards, S.J., Bryan, P., Marsh, S., Hobbs, P. and Mitchell, H., 2007. A robust surface matching technique for integrated monitoring of coastal geohazards. Marine Geodesy 30 (1-2), 109-123.

Mills, J.P., Buckley, S.J. and Mitchell, H.L., 2003. Synergistic fusion of GPS and photogrammetrically generated elevation models. Photogrammetric Engineering and Remote Sensing 69 (4), 341-349.

Mills, J.P., Buckley, S.J., Mitchell, H.L., Clarke, P.J. and Edwards, S.J., 2005. A geomatics data integration technique for coastal change monitoring. Earth Surface Processes and Landforms $30(6), 651-664$.

Mitchell, H.L., 1994. A comprehensive system for automated body surface measurement. International Archives of Photogrammetry, Remote Sensing and Spatial Information Sciences 30 (Part 5), 265-272.

Mitchell, H.L. and Chadwick, R.G., 1999. Digital photogrammetric concepts applied to surface deformation studies. Geomatica 53 (4), 405-414.

Pilgrim, L.J., 1996. Robust estimation applied to surface matching. ISPRS Journal of Photogrammetry and Remote Sensing 51 (5), 243-257. 
Rosenholm, D. and Torlegård, K., 1988. Three-dimensional absolute orientation of stereo models using digital elevation models. Photogrammetric Engineering and Remote Sensing 54 (10), 1385-1389.

Rosser, N.J., Petley, D.N., Lim, M., Dunning, S.A. and Allison, R.J., 2005. Terrestrial laser scanning for monitoring the process of hard rock coastal cliff erosion. Quarterly Journal of Engineering Geology and Hydrogeology 38 (4), 363-375.

Rousseeuw, P.J. and Leroy, A.M., 1987. Robust Regression and Outlier Detection, John Wiley \& Sons, New York.

Saye, S.E., van der Wal, D., Pye, K. and Blott, S.J., 2005. Beach-dune morphological relationships and erosion/accretion: an investigation at five sites in England and Wales using LIDAR data. Geomorphology 72 (1-4), 128-155.

Shrestha, R.L., Carter, W.E., Sartori, M., Luzum, B.J. and Slatton, K.C., 2005. Airborne laser swath mapping: quantifying changes in sandy beaches over time scales of weeks to years. ISPRS Journal of Photogrammetry and Remote Sensing 59 (4), 222-232.

Sithole, G. and Vosselman, G., 2003. Comparison of filtering algorithms. International Archives of Photogrammetry, Remote Sensing and Spatial Information Sciences 34 (Part 3/W13), 8 p.

Wager, T.D., Keller, M.C., Lacey, S.C. and Jonides, J., 2005. Increased sensitivity in neuroimaging analyses using robust regression. NeuroImage 26 (1), 99-113.

Wolf, P.R. and Dewitt, B.A., 2000. Elements of Photogrammetry with Applications in GIS, third ed. McGraw-Hill, New York.

World Bank, 1996. Guidelines for Integrated Coastal Zone Management, World Bank, Washington D.C., 16 p.

Würländer, R., Eder, K. and Geist, T., 2004. High quality DEMs for glacier monitoring - image matching versus laser scanning. International Archives of Photogrammetry, Remote Sensing and Spatial Information Sciences 35 (Part B7), 753-758. 


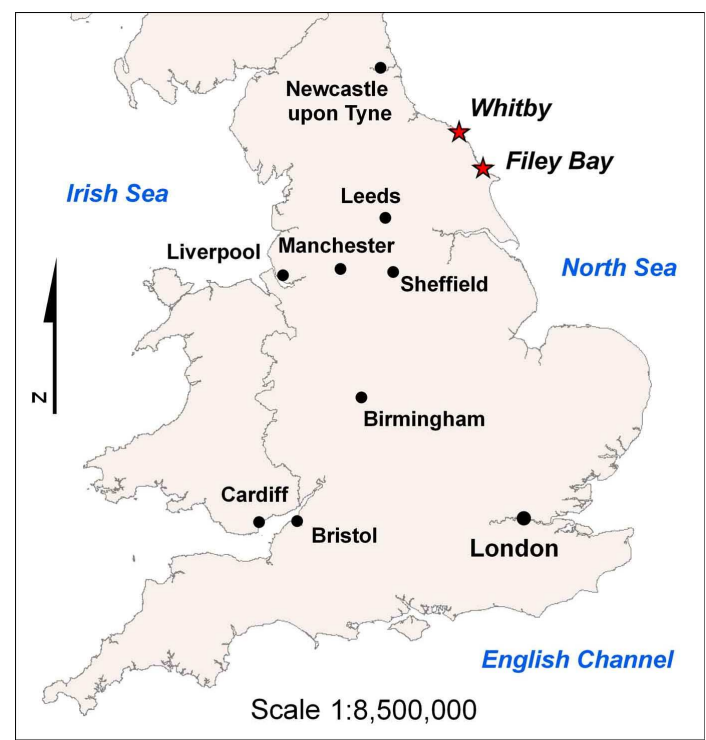

Figure 1. Location map

a)

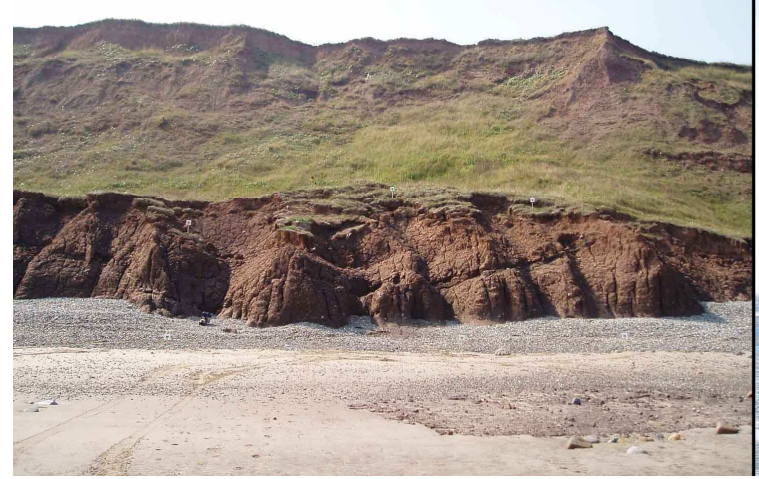

Figure 2. Test sites at Filey Bay (a) and Whitby Abbey Headland (b)

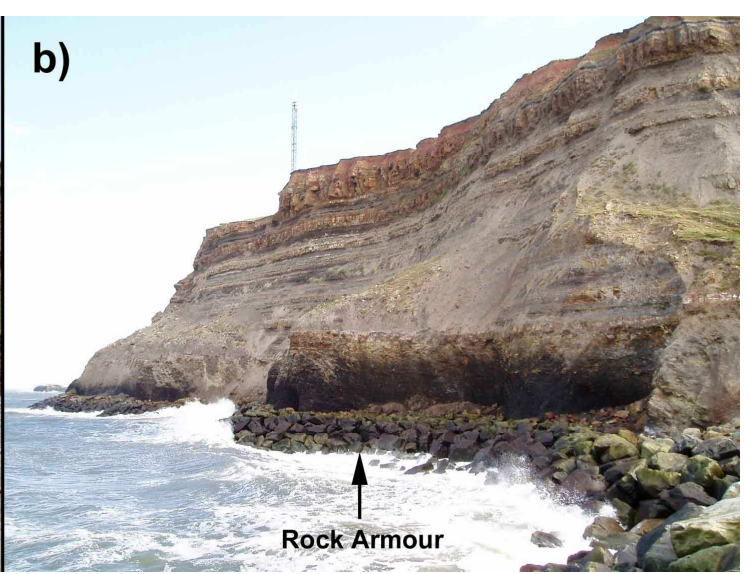

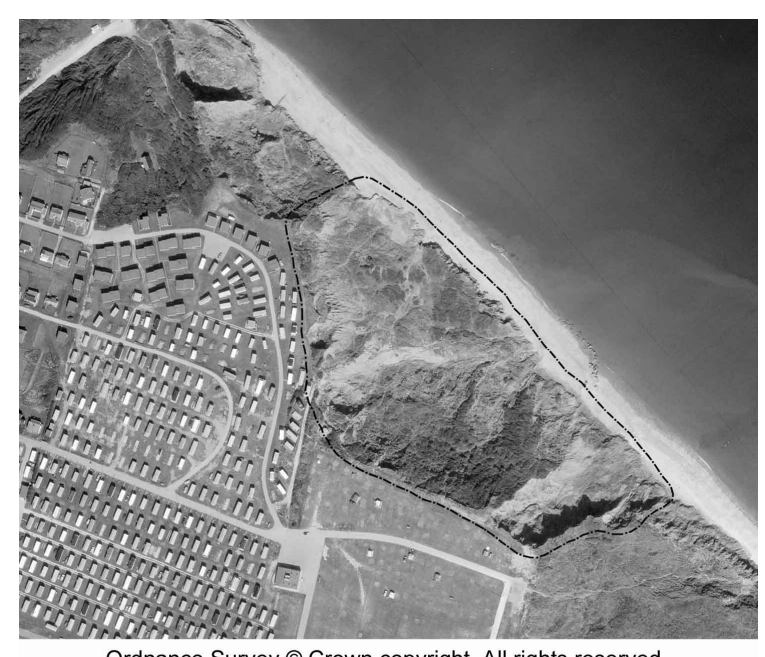

Ordnance Survey (c) Crown copyright. All rights reserved.

Figure 3. Test area (delineated) at southern end of Filey Bay, adjacent to caravan park 


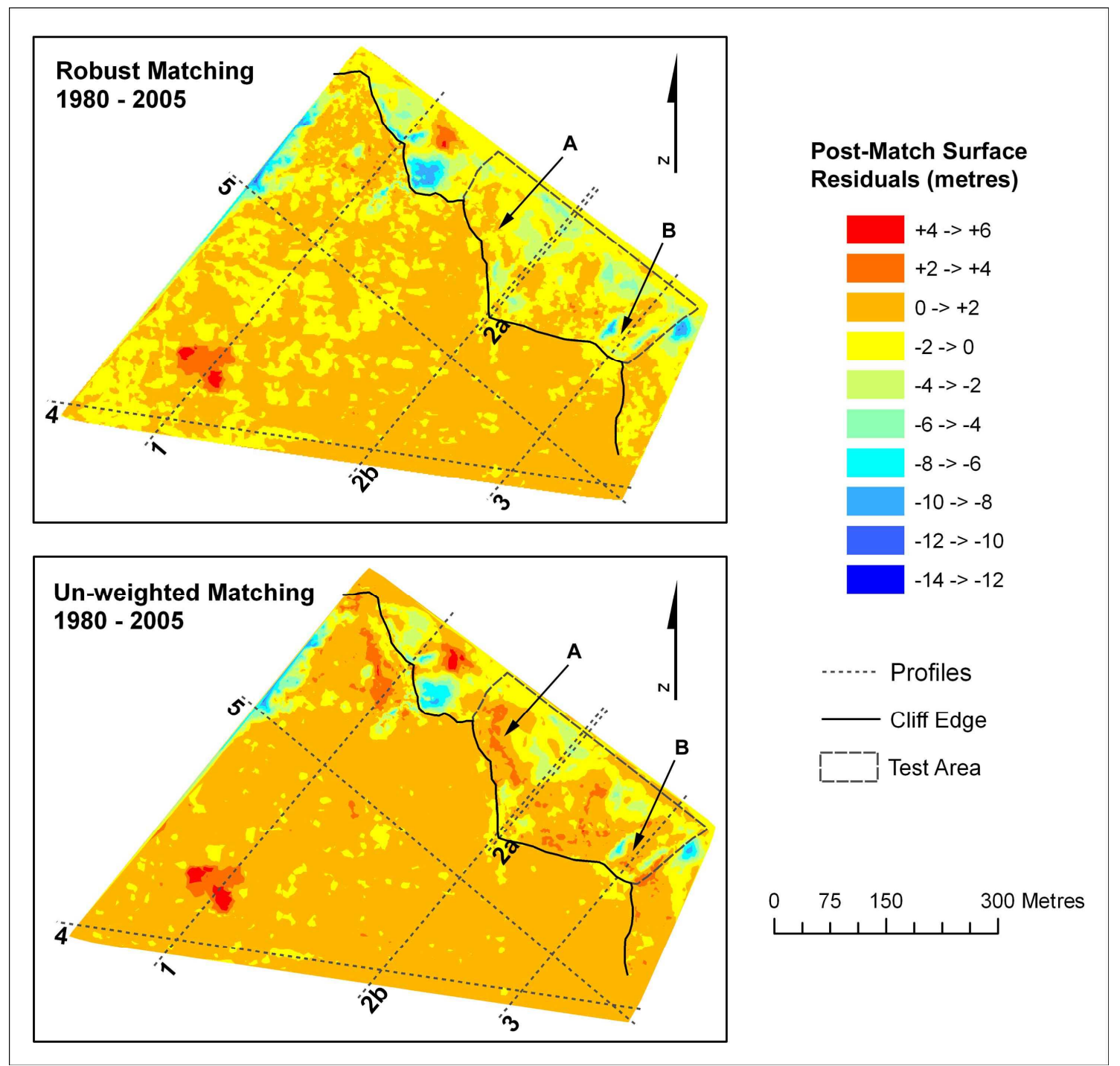

Figure 4. Post-match surface residuals for robust and un-weighted matching at Filey Bay test area 


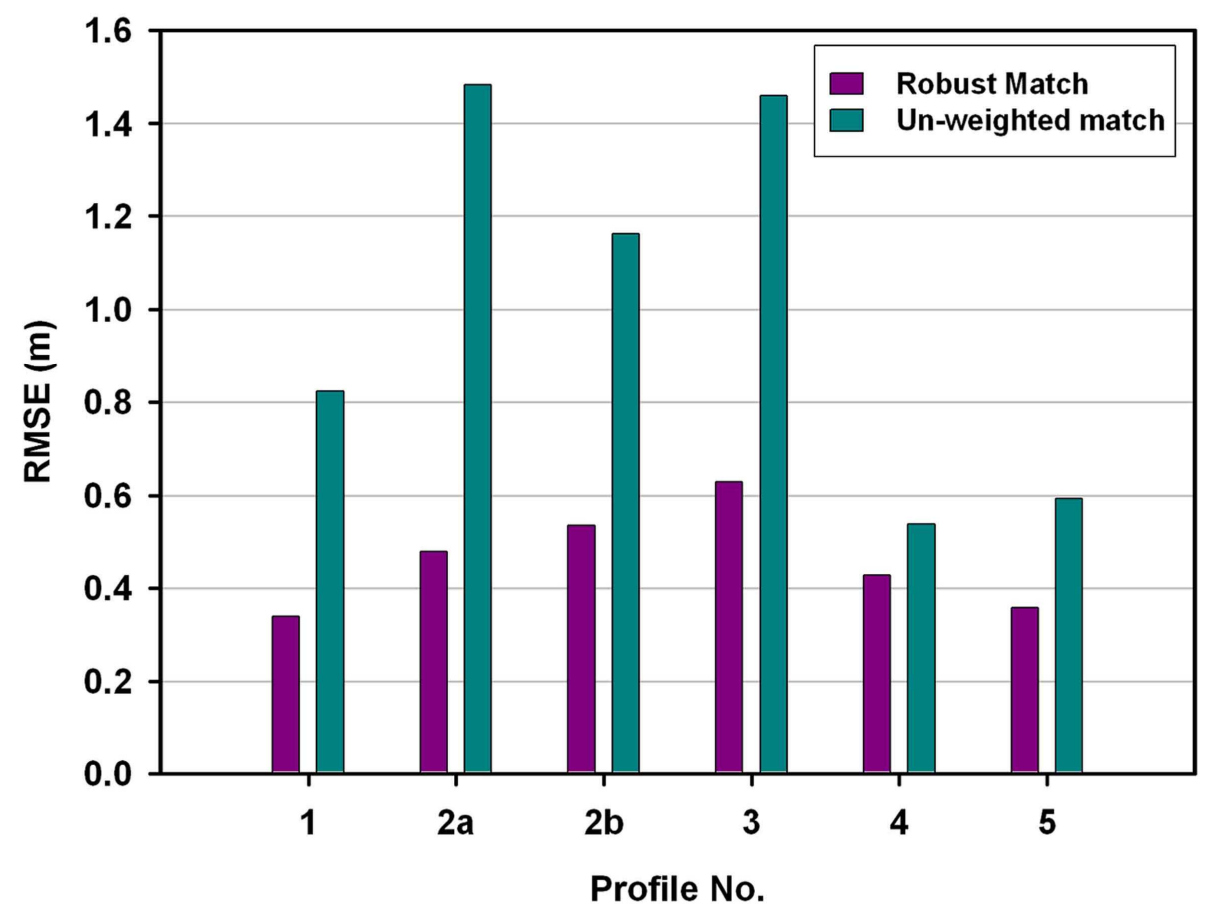

Figure 5. Profile accuracy statistics for robust and un-weighted matching solutions at Filey Bay test area

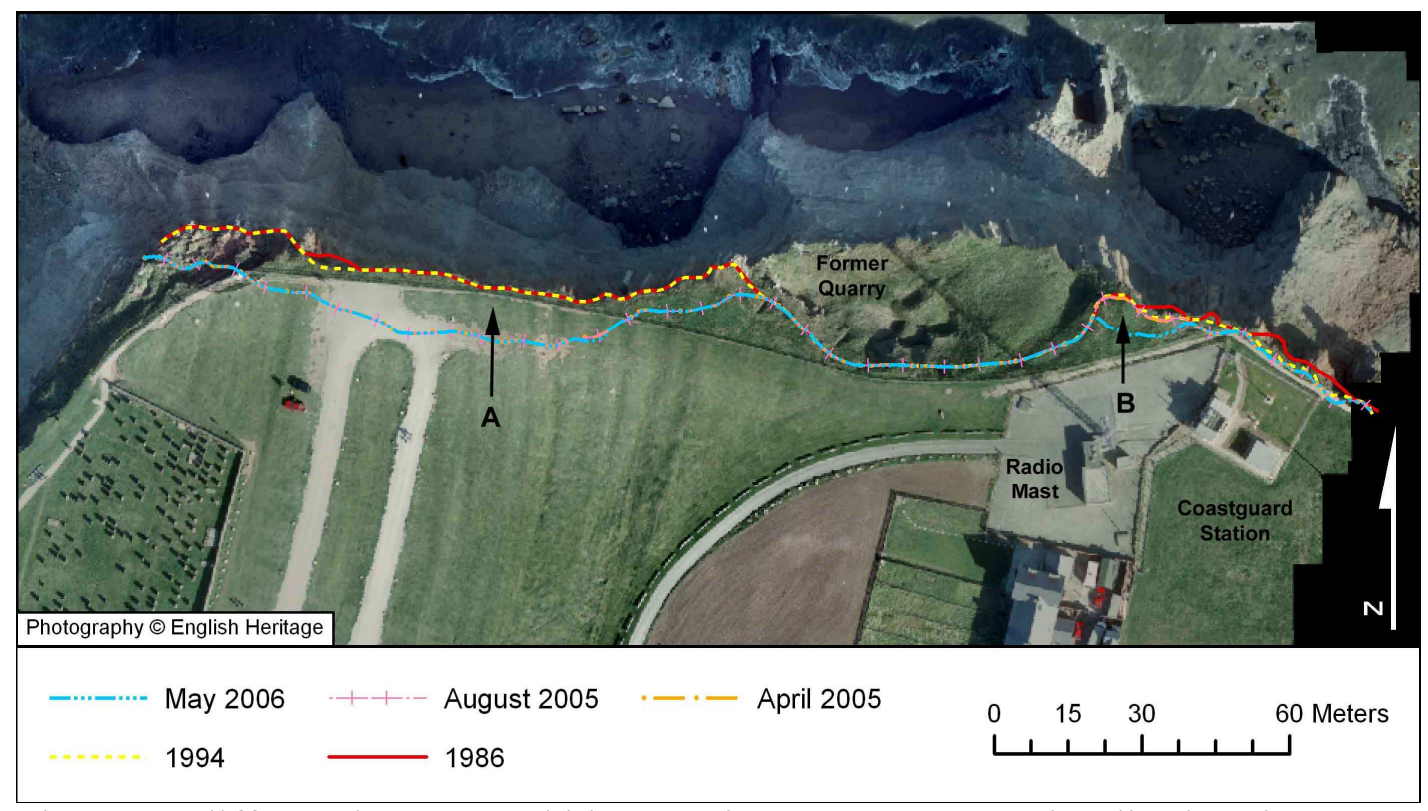

Figure 6. Cliff-top change at Whitby test site, 1986 to 2006, visualised against 1994 orthophoto. Area A indicates the location of the major 2000 cliff failure, while Area B is the site of a smaller, more recent failure 


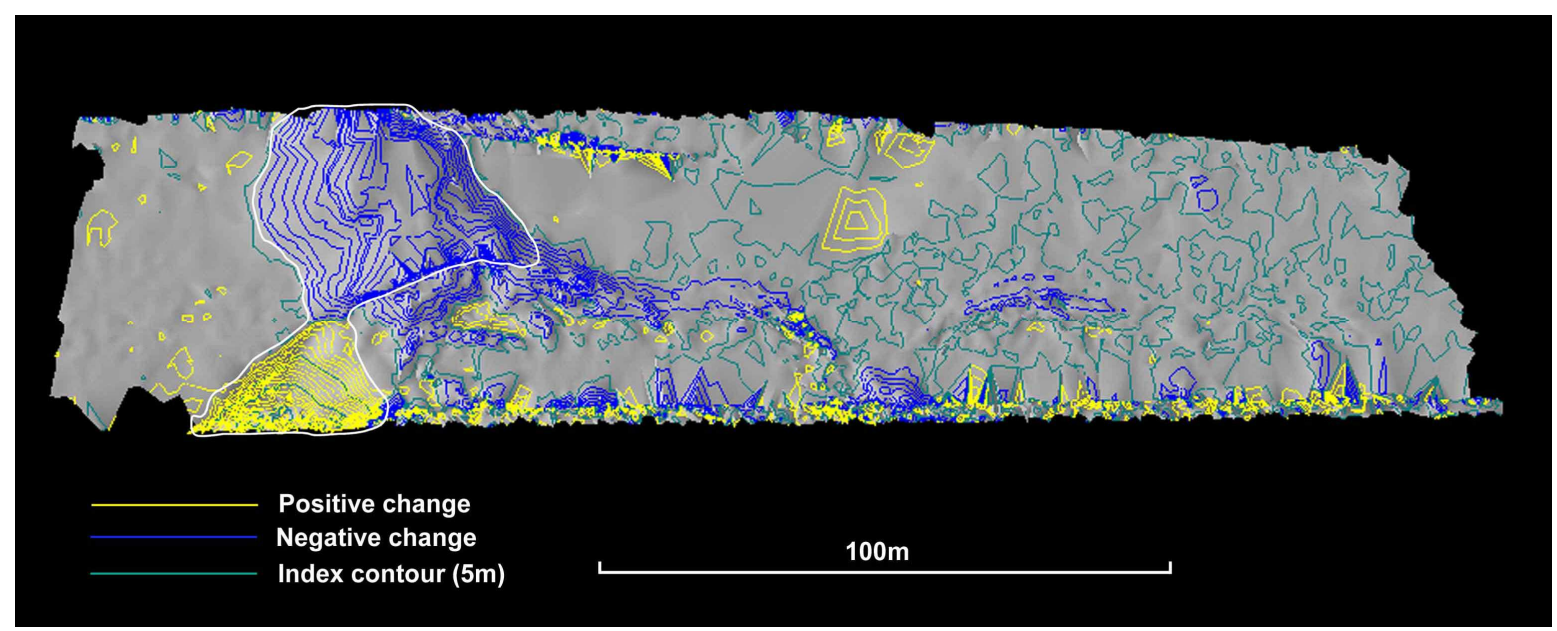

Figure 7. Changes across cliff face at Whitby test site between August 2005 and May 2006, with large failure area highlighted

\begin{tabular}{llll}
\hline Test Site & Year & Scale & Source \\
\hline Filey & 1980 & $1: 7800$ & Ordnance Survey \\
Whitby & 1986 & $1: 5300$ & Ordnance Survey \\
& 1994 & $1: 1600$ & English Heritage \\
\hline
\end{tabular}

Table 1. Details of archival photography acquired for the test sites

\begin{tabular}{llrrrrrr}
\hline \multicolumn{2}{l}{ Surface } & RMSE (m) & $\boldsymbol{\sigma}(\mathbf{m})$ & Mean (m) & Min. (m) & Max. (m) & $\begin{array}{c}\text { No. Check } \\
\text { Points }\end{array}$ \\
\hline 1994 & Reference & 0.130 & 0.132 & 0.023 & -0.250 & 0.260 & 17 \\
1986 & Pre-Match & 3.875 & 2.072 & -3.403 & -5.141 & -0.194 & 5 \\
& Un-weighted & 0.354 & 0.396 & -0.009 & -0.494 & 0.569 & 5 \\
& Robust & 0.150 & 0.165 & -0.046 & -0.194 & 0.099 & 4 \\
Apr. & Pre-Match & 0.347 & 0.152 & -0.314 & -0.625 & -0.098 & 18 \\
2005 & Un-weighted & 0.173 & 0.178 & 0.004 & -0.365 & 0.436 & 19 \\
& Robust & 0.156 & 0.159 & -0.010 & -0.365 & 0.386 & 20 \\
Aug. & Pre-Match & 0.324 & 0.206 & -0.255 & -0.654 & 0.126 & 18 \\
2005 & Un-weighted & & & No convergence & & \\
& Robust & 0.205 & 0.211 & -0.003 & -0.394 & 0.399 & 18 \\
May & Pre-Match & 0.162 & 0.155 & -0.060 & -0.374 & 0.142 & 18 \\
2006 & Un-weighted & 0.158 & 0.158 & -0.037 & -0.295 & 0.217 & 19 \\
& Robust & 0.148 & 0.139 & -0.060 & -0.295 & 0.167 & 19 \\
\hline
\end{tabular}

Table 2. Summary of quality statistics for surface matching at Whitby test site 